\title{
EFECTO DEL ÁREA MARINA PROTEGIDA DE LAS ISLAS DE VENTOTENE Y SANTO STEFANO SOBRE LA COMUNIDAD DE PECES
}

\author{
Enrique Ogallas* \\ Universidad de La Laguna
}

\section{RESUMEN}

El presente estudio pretende examinar el efecto de la reserva marina protegida de las islas de Ventotene y Santo Stefano, mediante el estudio de la biomasa de la comunidad de peces en las diferentes zonas de protección, Para ello se realizaron muestreos de abundancia y talla de peces en dos periodos de tiempo y en distintas localidades, dentro de la reserva. Las especies de peces encontradas se analizaron en diversos grupos con el objetivo de determinar el posible efecto de la reserva sobre estos grupos por separado. Por otro lado, y dado su importante papel socioeconómico, el mero fue analizado aparte. Los resultados indican que existen diferencias significativas en la biomasa de peces para las distintas zonas de la reserva estudiadas. La zona A de reserva integral presentó un mayor número y biomasa de especies comerciales bentónicas y el mero como unidad individual. Sin embargo, en las especies pelágicas, su biomasa vino determinada por la variabilidad estacional. El presente trabajo pone de manifiesto la utilidad del Área Marina Protegida de las islas de Ventotene y Santo Stefano para aumentar la biomasa de peces de interés comercial.

Palabras Clave: Área Marina Protegida, biomasa de peces, comunidad de peces, efecto reserva.

\author{
EFFECT OF THE MARINE PROTECTED AREA OF THE VENTOTENE \\ AND SANTO STEFANO ISLANDS ON THE FISH ASSEMBLAGES
}

\begin{abstract}
The present study aims to examine the effect of the protected marine reserve of the islands of Ventotene and Santo Stefano, by studying the biomass of the fish community in the different protection zones. To do this, we had to sample the abundance and measure the fish. This was done in two periods of time and in different locations, within the reserve. The fish species found were analyzed in various groups in order to determine the possible effect of the reserve on these groups separately. On the other hand, and given its important socioeconomic role, the mere, was analyzed separately. The results indicate that there are significant differences in fish biomass for the different areas of the reserve that we studied. Zone A of integral reserve presented a greater number and biomass of benthic commercial species and the grouper as an individual unit. However, in pelagic species, their biomass was determined by seasonal variability. The present work shows the usefulness of the Marine Protected Area of the islands of Ventotene and Santo Stefano to increase the biomass of the fish with commercial interest.
\end{abstract}

KeYwords: Community of fish, fish biomass, marine protected area, reserve effect. 


\section{INTRODUCCIÓN}

\section{Generalidades de las Áreas Marinas Protegidas}

Las Áreas Marinas Protegidas (AMPs) han tenido, a escala global, un desarrollo fundamental en las últimas décadas puesto que son consideradas como un instrumento eficiente para la protección de la biodiversidad, la conservación del ambiente marino y como modelo para el estudio de las poblaciones marinas en ausencia de la pesca (Massaro, 2009). El éxito de éstas se mide en relación al cumplimiento de lo planificado en los objetivos de gestión, teniendo en cuenta que este cumplimiento dependerá de las especies protegidas o del tiempo con respecto al establecimiento del AMP (Mosqueira et al., 2000; Claudet et al., 2006). Los efectos positivos de las AMPs se ven claramente en el aumento de la abundancia y biomasa de la comunidad de especies marinas, aumento de la proporción de individuos más grandes y longevos (Côté et al., 2001) e incluso en el aumento de los rendimientos de pesca local en zonas adyacentes (García-Charton et al., 2008).

En el Mediterráneo, el número de AMPs ha aumentado desde el primer parque nacional marino de Port-Cros en 1963 (Francour et al., 2001; Fraschetti et al., 2005). En general, el tipo de AMP más extendidas son aquellas donde las actividades humanas están reguladas, a fin de conciliar las necesidades de conservación con las del desarrollo sostenible (Badalamenti et al., 2000). Las AMPs en las cuales cualquier forma de actividad antrópica está prohibida, son bastante raras en el Mediterráneo y suelen ser de pequeño tamaño. En el caso de Italia, la legislación nacional prevé la puesta en funcionamiento de AMPs con objetivos tanto naturalistas como socioeconómicos $\mathrm{y}$, por lo tanto, se suelen sectorizar en niveles de protección diferentes: Zona «reserva integral» A, Zona reserva «general» B y Zona de reserva "parcial» C (Di Donato, 2008).

Desde un punto de vista ecológico, la alteración de los ecosistemas marinos litorales por la sobrepesca de los depredadores de mayor tamaño, genera un efecto cascada sobre las cadenas tróficas (Tegner \& Dayton, 2000; Shears \& Babcock, 2002), desequilibrando el ecosistema marino y generando sistemas empobrecidos con poca capacidad para generar beneficios económicos. En este sentido, varios estudios han explorado el efecto del cese de la pesca dentro de las reservas marinas y han descubierto que, en general, la abundancia y biomasa de las poblaciones de peces explotadas son mayores dentro de las áreas protegidas comparado con las áreas cercanas a la reserva, tanto en el Mediterráneo (Bell, 1983; García-Rubies \& Zabala, 1990; Francour, 1994; Harmelin et al., 1995) como en otras zonas del Atlántico (García-Charton et al., 2008).

* Ecología de Comunidades Marinas y Cambio Climático. Departamento de Biología Animal, Edafología y Geología (U.D. Ciencias Marinas), Facultad de Ciencias (Biología). Corresponding author: ogallas.moliz@gmail.com. 


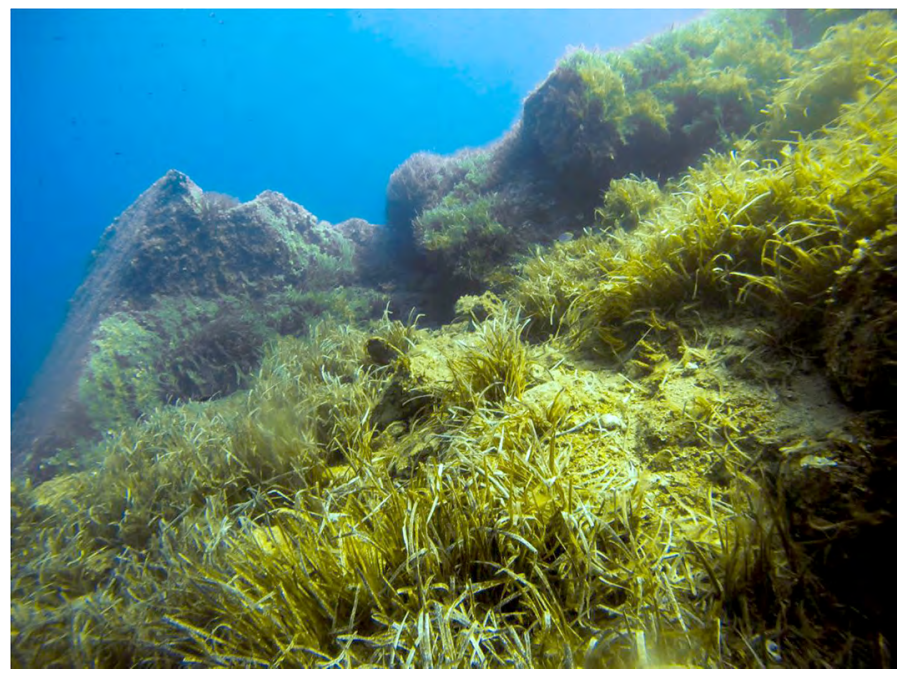

Figura 1. Fondo rocoso recubierto por Posidonia oceanica observado durante los muestreos en la zona B situada en la isla de Santo Stefano, que desciende hasta los 40 metros de profundidad.

\section{AMP de las islas de Ventotene y Santo Stefano}

El Área Marina Protegida de Ventotene y Santo Stefano (AMP de VSS) comprende las aguas entre ambas islas, con una extensión de unas 2800 ha de superficie marina y $10 \mathrm{~km}$ de costa. Éstas junto con Ponza, Zannone y Palmarola constituyen el archipiélago Pontino, caracterizado por presentar una geografía de origen volcánico. Ventotene y Santo Stefano son las islas más recientes del archipiélago, formándose hace 800000 años.

Este AMP fue declarada el 12 de diciembre de 1997 por el Ministerio de Ambiente, quedando reflejada en la ley de áreas marinas protegidas n. 394/91. Dos años más tarde, el 11 de mayo de 1999 fue proclamada por el decreto del ministerio como Reserva Natural Terrestre.

Estas dos islas se caracterizan por presentar unos fondos marinos muy heterogéneos, lo que favorece el asentamiento de una gran diversidad de poblaciones bentónicas. La presencia de numerosos veriles, cavidades y pequeñas cuevas permiten la existencia de organismos esciáfilos a pocos metros de profundidad. Las praderas de la fanerógama marina Posidonia oceanica son también de gran importancia (figura 1), ya que alcanzan profundidades de más de 40 metros gracias a la transparencia del agua. Los numerosos deslizamientos de tierra, típicos de los riscos volcánicos, son lugares importantes para el reclutamiento de especies de peces. Otro aspecto importante a destacar es el paso estacional de las grandes especies pelágicas, como Trachinotus ovatus, Sphyraena viridensis o Seriola dumerili (figura 2), así como grupos de cetáceos (Ministerio de Marina Mercantil, 1990; Di Stefano, 2004). 


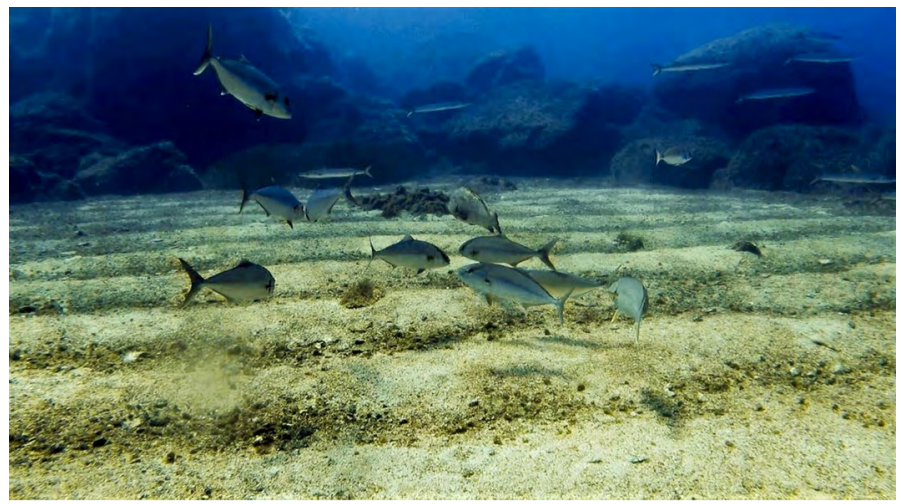

Figura 2. Las zonas abiertas son zonas de paso utilizadas comúnmente por especies de peces pelágicos, como medregales o barracudas. Sitio de muestreo La Molara, Zona A.

1.1. Objetivo del trabajo: Funcionalidad del AMP a través del «efecto reserva» en las distintas zonas de protección.

La necesidad de creación de un AMP surgió por la creciente evidencia de que la explotación excesiva de los recursos pesqueros tiene un impacto claro sobre las especies de interés comercial y una desestructuración de las comunidades y ecosistemas marinos (Jackson et al., 2001), con efectos perjudiciales sobre éstos (Worm et al., 2006; Cattaeo-Vietti et al., 2011).

La evaluación del llamado "efecto de reserva» consiste en el estudio del aumento potencial en densidad y tamaño y / o biomasa (entendido como 'peso fresco' por unidad de área de muestra) de las especies objetivo de la pesca dentro de un AMP en comparación con las áreas de control abiertas a la pesca, de acuerdo con unos planes de muestreo apropiados (Guidetti, 2002) (figura 3).

En el presente estudio, nos centraremos en comprobar si se cumple el efecto reserva, comparando la abundancia y biomasa de peces de las distintas zonas de protección, haciendo un especial hincapié en especies de interés comercial.

\section{MATERIAL Y MÉTODOS}

\section{Características de la RESERVA MARINA}

De acuerdo con el decreto institucional, los objetivos de las AMPs son:

- La protección ambiental del área marina interesada.

- La valoración de los recursos biológicos y geomorfológicos de la zona. 


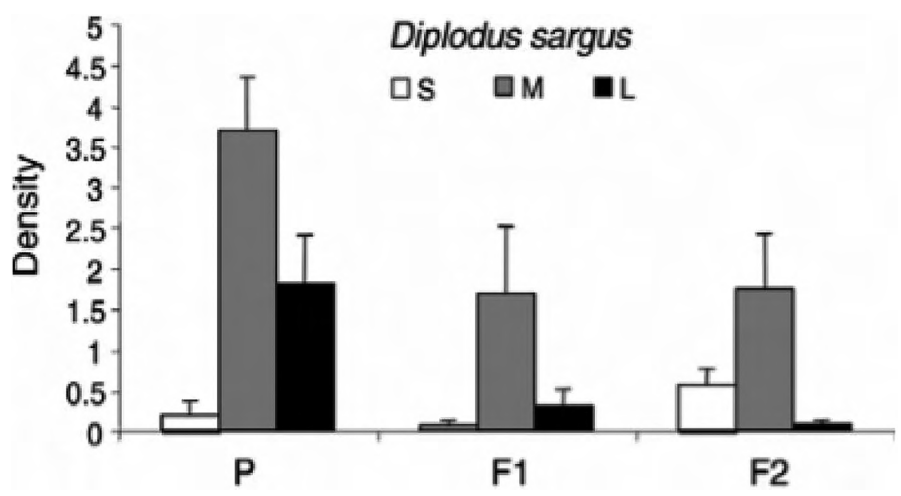

Figura 3. Ejemplo de 'efecto de reserva' (ER). Densidad media ( \pm SE, número de individuos $125 \mathrm{~m}^{2}$ ) de Diplodus sargus, en sitios protegidos (P) del Área Marina Protegida de Miramare (Trieste) y desprotegido (F1, F2), en relación con el tamańo del pez (S: pequeño = pequeño; M: medio = mediano; L: grande = grande). Guidetti et al., 2005).

- La divulgación y difusión del conocimiento de la ecología y de la biología de los ambientes marinos y costeros del área natural protegida y de las características ambientales y geomorfológicas de la zona.

- La realización de programas de carácter educativo para la mejora de la cultura general de la ecología y de la biología marina.

- La realización de programas de estudio y de búsqueda científica en los sectores de la ecología, de la biología marina y para la protección del medio ambiente.

- La promoción de un desarrollo socio-económico compatible con la relevancia natural y paisajística del área, con la inclusión de actividades tradicionales locales presentes (Marino, 2011).

La superficie del AMP está dividida en zonas caracterizadas por el tipo de protección que presentan. El AMP de VSS está sectorizada en tres áreas: La Zona A o reserva integral, la Zona $\mathrm{B}$ o reserva general y la Zona $\mathrm{C}$ o reserva parcial (Tunesi \& Diviacco, 1993) (figura 4).

La zona A es el corazón de la reserva, en ella está prohibido todo tipo de actividad que pueda causar daño o perturbación en el medio marino, salvo aquellas relacionadas con su gestión y la investigación. Presenta un área de 410 ha.

La zona $\mathrm{B}$ es una zona donde se permite una serie de actividades con un impacto muy reducido, como por ejemplo buceo, bańo, visitas guiadas, amarre y fondeo en zonas específicas o la navegación a baja velocidad. Presenta un área de 1600 ha.

Por último, la zona $\mathrm{C}$ es un área de amortiguamiento entre las zonas de mayor importancia naturalista y los sectores fuera del AMP. En ella están permi- 


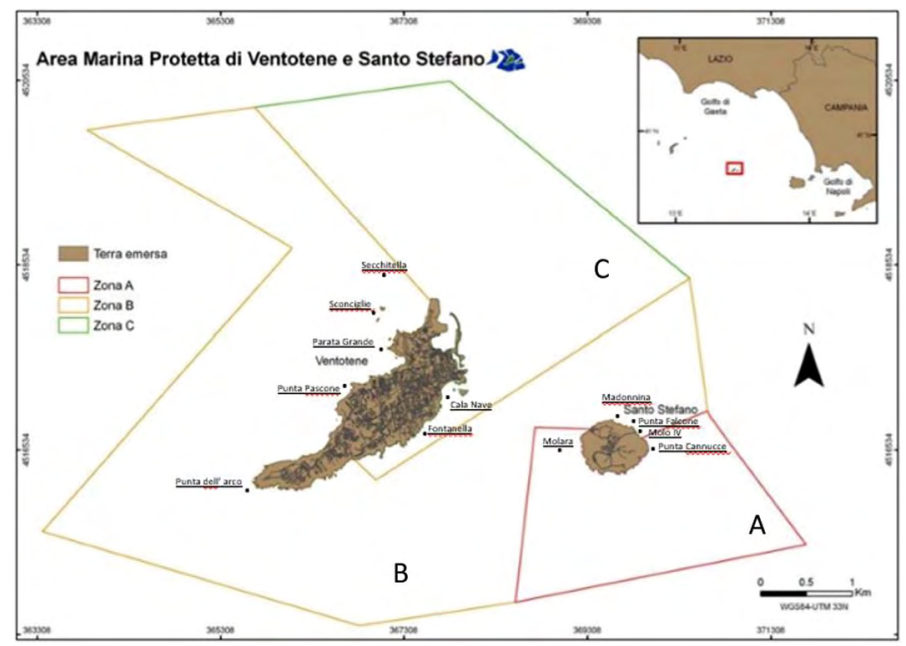

Figura 4. Zonación de las tres zonas del AMP-VSS (A, B y C) y localidades de muestreo.

tidas actividades con un impacto moderado, como la navegación de embarcaciones a motor, amarre, fondeo o incluso la pesca deportiva (Di Donato, 2008). Presenta un área de 789 ha.

El objetivo de la Zona $\mathrm{B}$ y de la Zona $\mathrm{C}$ es combinar las necesidades de conservación con el uso sostenible de los recursos marinos.

A modo de resumen, ver la tabla 1 , donde se especifican los usos permitidos en cada uno de los sectores del AMP.

\begin{tabular}{|c|c|c|c|c|c|c|c|}
\hline \multicolumn{8}{|c|}{$\begin{array}{l}\text { TABLA 1. REGLAMENTO DE LAS ACTIVIDADES REGULADAS DENTRO DEL AMP DE VSS. } \\
\text { L: ACTIVIDAD SUJETA A LIMITACIONES. A: ACTIVIDAD CONSENTIDA CON } \\
\text { AUTORIZACIÓN. S: ACTIVIDAD CONSENTIDA. N: ACTIVIDAD NO CONSENTIDA }\end{array}$} \\
\hline 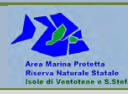 & NAVEGACIÓN & Amarre & BAÑo & $\begin{array}{c}\text { Pesca } \\
\text { PROFESIONAL }\end{array}$ & $\begin{array}{c}\text { Pesca } \\
\text { Deportiva }\end{array}$ & $\begin{array}{l}\text { VISITAS } \\
\text { GUIADAS }\end{array}$ & $\begin{array}{l}\text { BUCEO CON } \\
\text { EQUIPO PESADO }\end{array}$ \\
\hline ZONA A & $\mathrm{L} / \mathrm{A}$ & $\mathrm{N}$ & $S$ & $\mathrm{~N}$ & $\mathrm{~N}$ & $\mathrm{~N}$ & L/A \\
\hline ZONA B & $\mathrm{L} / \mathrm{A}$ & $\mathrm{L}$ & $S$ & $\mathrm{~L} / \mathrm{A}$ & $\mathrm{L} / \mathrm{A}$ & A & A \\
\hline ZONA C & S & $\mathrm{L}$ & S & $\mathrm{L} / \mathrm{A}$ & L/A & A & S \\
\hline
\end{tabular}

Para el estudio de las poblaciones de peces, la distribución de tamaños y los efectos de la protección, se realizaron un total de 77 muestreos en el interior del AMP de VSS durante el verano de 2017 (junio-septiembre), repartidos en las diferentes zonas del AMP. Como se observa en la figura 4, los puntos de muestreo elegidos fueron: en la zona $\mathrm{C}$ las localidades «Cala Nave» $\mathrm{y}$ «Fontanella»; en la zona B «Punta Pascone», «Shecchitella», «Sconciglie», «Parata Grande», «Punta dell'Arco», «Molo IV», «Punta Falcone» $\mathrm{y}$ "Madonnina»; mientras que en la zona A se eligieron las localidades «Punta Cannucce» $\mathrm{y}$ «Molara». 


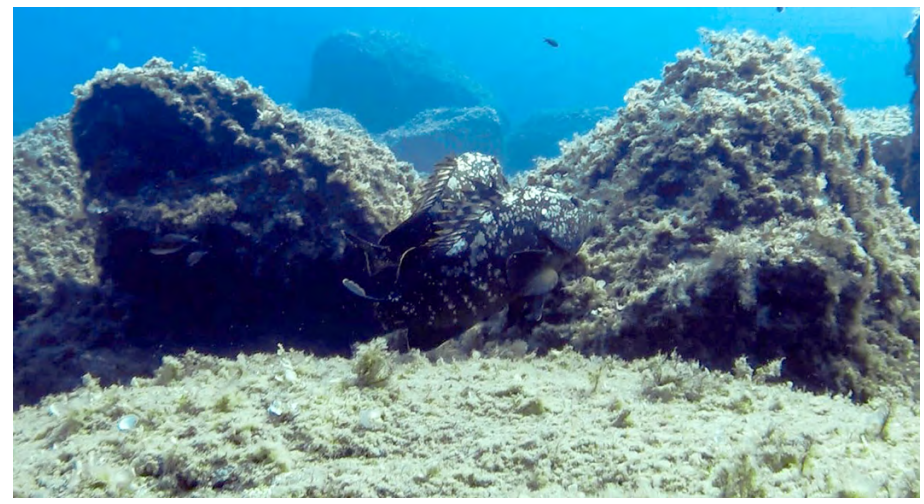

Figura 5. Dos ejemplares de E. marginatus camuflados sobre las rocas del fondo de la localidad de Molo Iv, Santo Stefano.

Cada una de las localidades fue muestreada en dos tiempos diferentes para tener en cuenta la posible variabilidad temporal dentro de la misma época del año.

\section{ORgaNismos y COMUNIDADES PRESENTES EN LOS FONDOS DEL AMP-VSS}

La isla de Ventotene y sobre todo la de Santo Stefano se caracterizan por presentar ambientes con fondos rocosos que alcanzan profundidades de cincuenta metros a una corta distancia de la costa. Estas rocas superpuestas forman cavidades donde especies sedentarias encuentran en ellas refugio. Este es el caso del mero, Epinephelus marginatus. Esta especie es uno de los depredadores top más grandes que habitan en estos fondos ricos en refugios rocosos (figura 5). E. marginatus tiene una importancia considerable para la pesca deportiva y las pesquerías comerciales locales, si bien es muy vulnerable a la sobreexplotación. Es de destacar que actualmente, algunas subpoblaciones son custodiadas en áreas marinas protegidas, donde esta especie ha mostrado aumentos significativos en la densidad y el tamańo medio después del cese de la pesca (Sánchez-Lizaso et al., 2000; Reñones et al., 2005).

También es característico ver grandes praderas de Posidonia oceanica en casi todos los fondos. En las partes más superficiales del submareal encontramos estas praderas intercaladas con extensiones de arena y sustrato rocoso en forma de afloramientos o cantos rodados (figura 6) junto con la presencia de biocenosis de algas fotófilas, como Halimeda tuna y Udotea petiolata, y especies de algas pardas dominantes como Cystoseira stricta o Padina pavonica y, en las zonas de sombra, es característico encontrarse formaciones de Astroides calycularis y Parazoanthus axinellae (figura 7).

En la zona media de los muestreos en el submareal, encontramos fondos rocosos como los mencionados anteriormente y arenales rodeados de praderas de Posidonia oceanica (figura 8). Presenta una biocenosis de especies esciófilas hasta los 


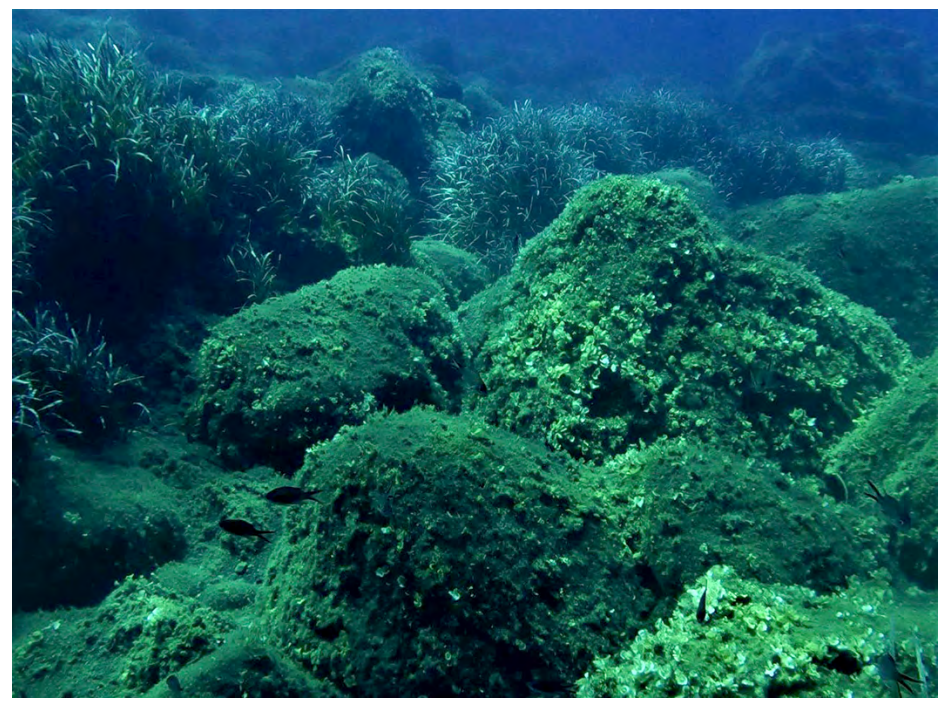

Figura 6. Submareal poco profundo con fondo de rocas, arena y Posidonia oceanica.

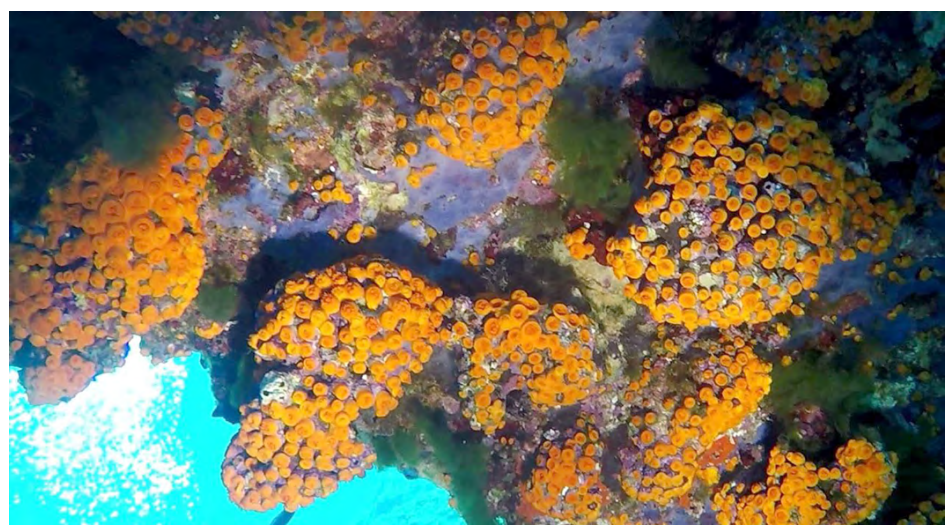

Figura 7. Pared recubierta por Astroides calycularis.

30-35 metros, a partir de la cual, se encuentra la biocenosis del coralígeno, caracterizada por algas pardas y rojas, así como briozoos incrustantes. Algunos organismos a destacar de éstas formaciones son de Myriapora truncata, Pentapora fascialis o Sertella septentrionalis. En los fondos de Santo Stefano se pueden encontrar también las gorgonias Paramuricea clavata o Eunicella cavolini (figura 9). 


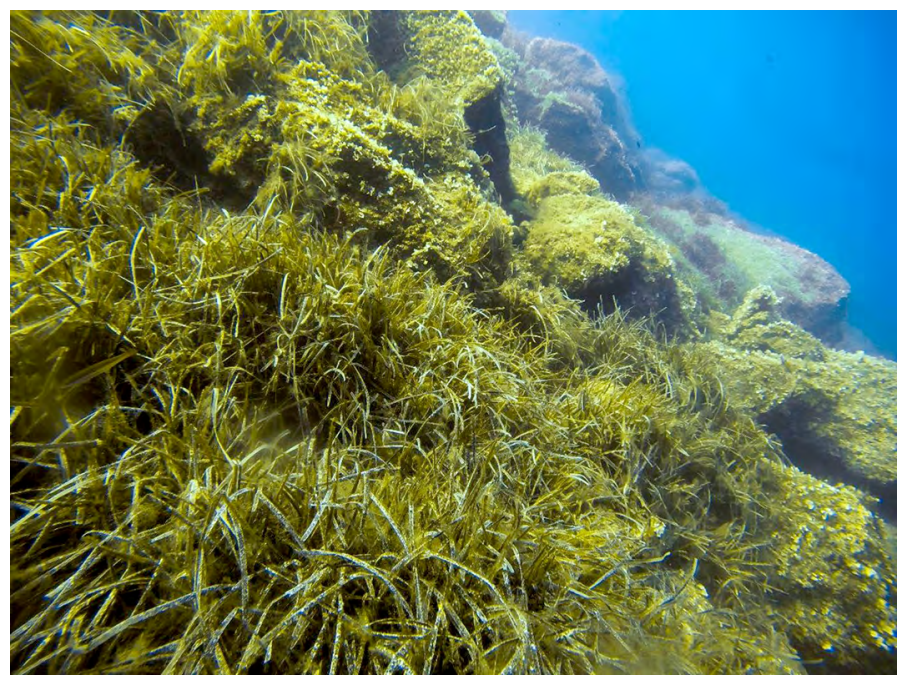

Figura 8. Fondos rocosos que descienden hasta una profundidad de 50 metros.
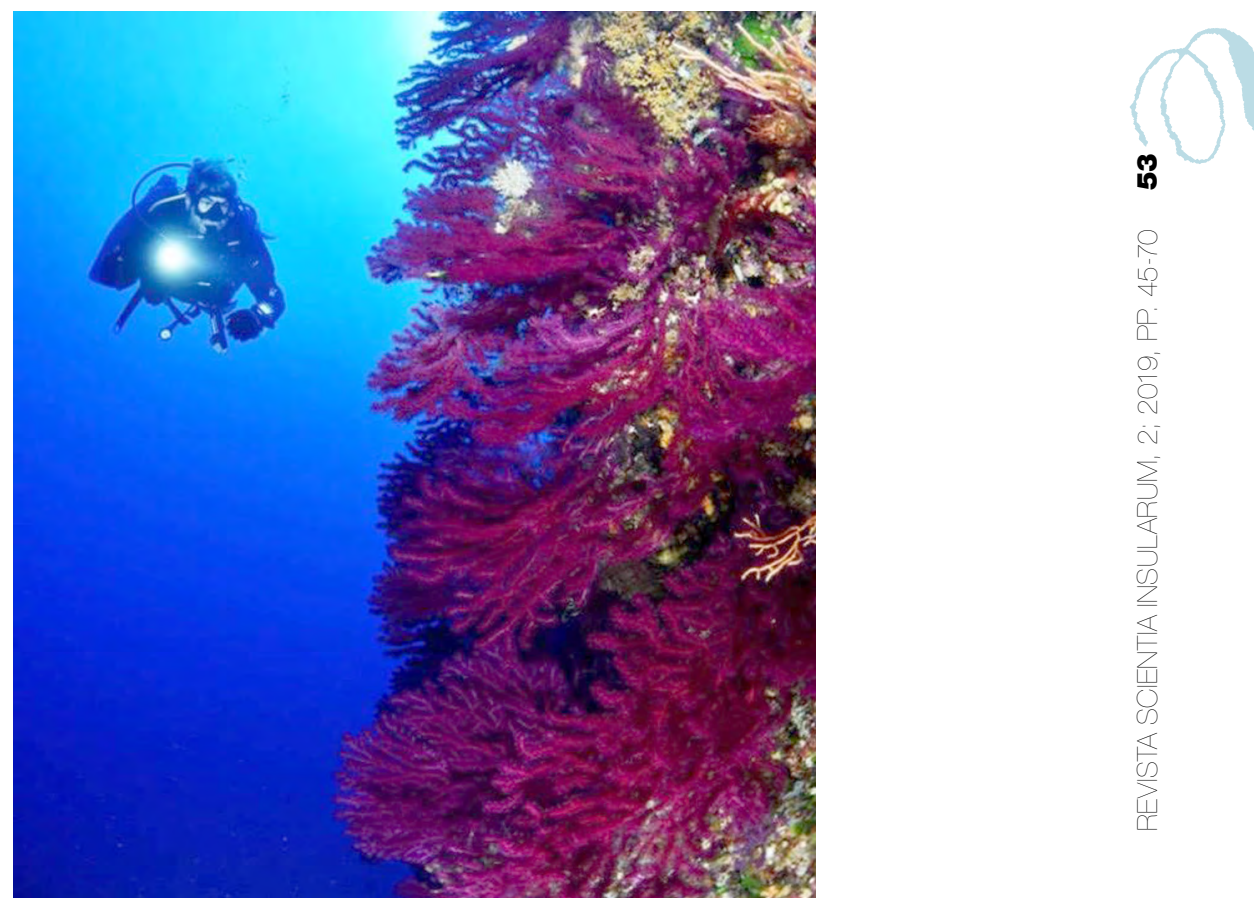

Figura 9. Pared de gorgonias de la especie Paramuricea clavata, a 30 metros de profundidad en la localidad de Punta Cannucce, Santo Stefano. 


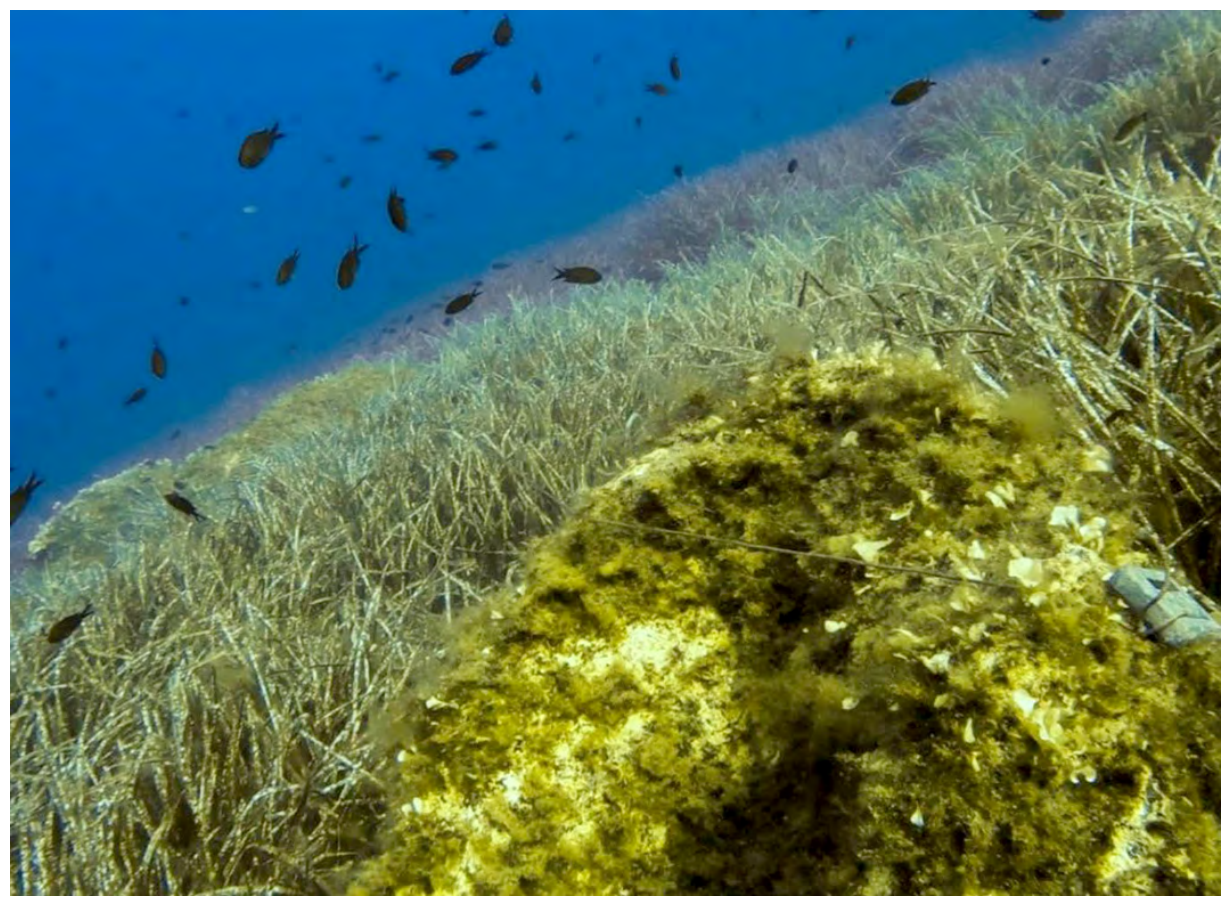

Figura 10. Método de muestreo UVC de punto fijo utilizado para los muestreos en la localidad de Punta Falcone, Santo Stefano. En la fotografía se puede observar el transecto utilizado para calibrar el radio de la circunferencia imaginaria.

\section{Metodología de Muestreo}

La metodología de Underwater Visual Census (UVC), es una técnica visual bajo el agua que se introdujo en la década de 1950, pero su gran desarrollo tuvo lugar durante la década de 1970 (Harmelin-Vivien et al., 1975) en el campo de la investigación de peces en el Parque nacional francés de Port-Cros. Este tipo de metodología no destructiva permite observar los organismos «in situ» sin necesidad de extracción, lo que reduce el tiempo y los costos necesarios para ciertos tipos de estudios, como el presente.

\section{Método UVC de punto fijo}

En este trabajo, para producir el menor daño posible a las comunidades bentónicas, se utilizó un método visual 'in situ' para el recuento de peces en aguas someras (Bardach, 1959; Mapstone \& Ayling, 1998). Esta técnica de recuento subacuático es denominada «Punto fijo/Circular» y fue descrita por Bortone et al., (1989) y con- 


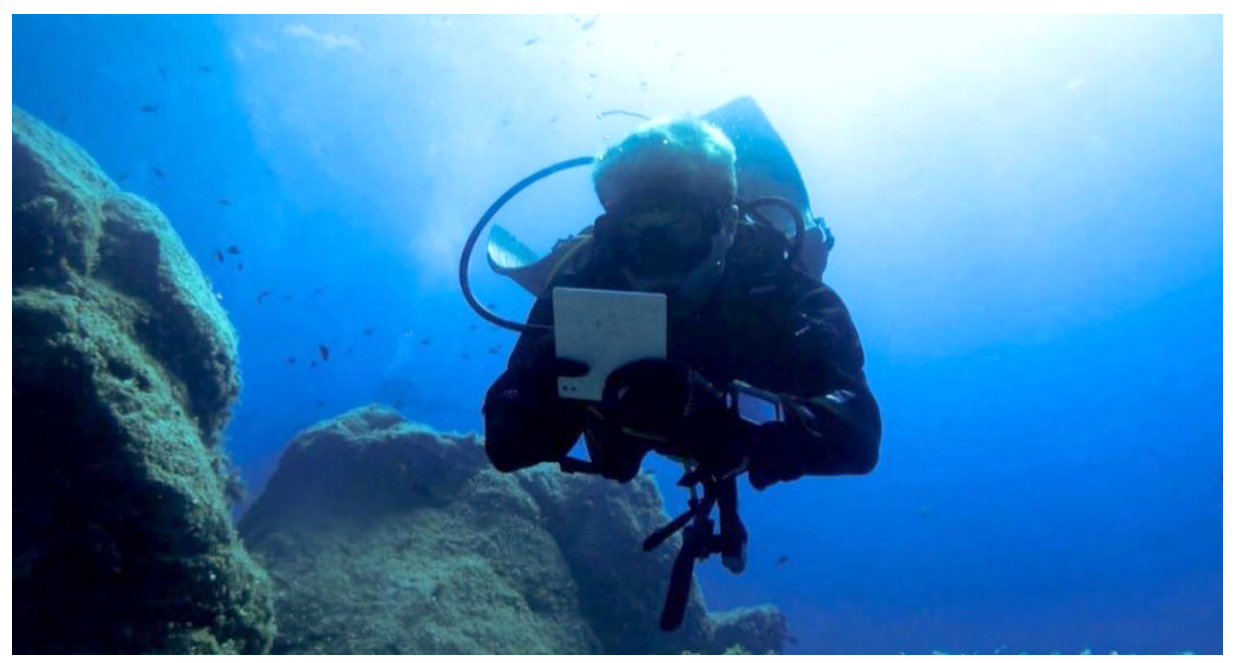

Figura 11. Buceador realizando los muestreos.

siste, mediante un equipo de buceo autónomo, situarse en un punto fijo y observar alrededor de un cilindro imaginario de 5,6 metros de radio, que se extiende desde la superficie hasta el fondo y que representa una superficie de $100 \mathrm{~m}^{2}$ en total, medida muy útil para el muestreo de una gran diversidad de peces con distintos tamaños (Bortone et al., 1991) (figura 10). El observador va girando sobre ese punto central y se anotan en una pizarra de PVC las especies que entran dentro del cilindro, registrando su abundancia y talla durante 5 minutos (figura 11). También se recogen otros datos como la fecha, hora, profundidad, temperatura mínima del agua (proporcionadas por un ordenador de buceo) y tipo de ambiente para cada muestreo. El último minuto del muestreo se emplea para la búsqueda de especies crípticas.

En algunas ocasiones, los cardúmenes muy numerosos pueden ser un problema a la hora del recuento, nosotros seguimos las recomendaciones de Brock (1954), es decir, si el banco entra dentro del cilindro se procede a contabilizar una porción y se estima el conjunto al completo. Por otro lado también es importante tener en cuenta que sólo se realiza el recuento una sola vez de los peces que entran repetidas veces durante el tiempo de muestreo.

Para el reconocimiento de especies, primero se hicieron algunos muestreos de prueba y, con la ayuda de fishbase (www.fishbase.org) y catálogos especializados del mediterráneo, se logró identificar a los peces encontrados en los muestreos.

Los tamaños de las especies se registraron utilizando siete clases de talla $(<5$, [5-10], (10-20], (20-30], (30-50], (50-75] y >75) ó (<5, [5-10], [11-20], [21-30], [3150], [51-75] y >75), correspondientes a un tercio del tamaño máximo reportado en la literatura para las especies registradas (Francour, 1999). 
Con los datos recogidos en cada muestreo se procedió a realizar tres análisis multivariantes por permutaciones, PERMANOVA y uno univariante, PERANOVA (Anderson et al., 2005). Se sometieron a análisis las variables biomasa total de peces, biomasa de especies comerciales bentónicas, biomasa de especies comerciales pelágicas y biomasa de Epinephelus marginatus.

Para cada uno de los casos analizados se aplicó el siguiente diseño:

- Zona: factor fijo con tres niveles: Integral, General y Parcial.

- Localidad: factor aleatorio anidado en zona. Cada uno de los puntos de muestreo (Cala Nave, Fontanella, Secchitella, Molara...).

- Tiempo: factor aleatorio. Tiempo 1: junio-julio, tiempo, y tiempo 2: agosto-septiembre.

Los resultados fueron representados mediante un análisis de ordenación de componentes principales (PCO) y gráficas de barras-error.

\section{RESULTADOS}

\section{ANÁLISIS DESCRIPTIVO}

Se encontraron un total de 23825 individuos repartidos en 21 familias, 38 géneros y 56 especies (ver tabla 2).

Las especies se clasificaron en cuatro grupos: comunidad total de peces, especies de interés comercial bentónicas (indicadas con un * en la tabla 2), especies de interés comercial pelágicas ${ }^{\times}$) y Epinephelus marginatus como especie individual, por ser una especie emblemática para la zona.

Atendiendo a los resultados de las abundancias, las especies que obtuvieron un mayor número de individuos fueron, en primer lugar Chromis chromis, con un recuento total de 17445 individuos; seguido de Anthias anthias, 982 individuos; Thalasoma pavo, 979 individuos; y Coris julis con 798 individuos. 


\begin{tabular}{|c|c|c|c|c|c|}
\hline 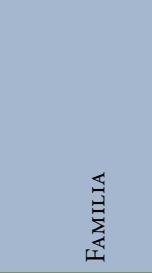 & 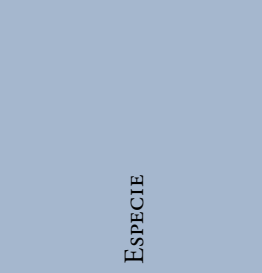 & $\begin{array}{l}\approx \\
0 \\
5 \\
5 \\
\end{array}$ & 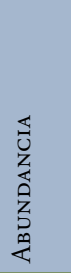 & 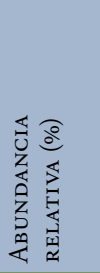 & 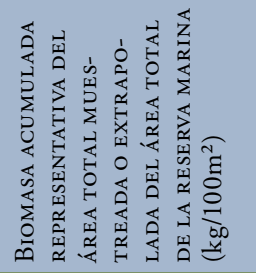 \\
\hline Apogonidae & Apogon imberbis & Linnaeus, 1758 & 293 & 1,230 & 4,224 \\
\hline Atherininae & Atherina boyeri & Risso, 1810 & 240 & 1,007 & 3,536 \\
\hline Blenniidae & $\begin{array}{l}\text { Parablennius pili- } \\
\text { cornis }\end{array}$ & Cuvier, 1829 & 14 & 0,059 & 0,202 \\
\hline Blenniidae & Parablennius rouxi & Cocco, 1833 & 3 & 0,013 & 0,009 \\
\hline Bothidae & Bothus podas & Delaroche, 1809 & 1 & 0,004 & 0,077 \\
\hline Carangidae & Seriola dumerili ${ }^{\times}$ & Risso, 1810 & 11 & 0,046 & 15,752 \\
\hline Carangidae & Trachinotus ovatus & Linnaeus, 1758 & 1 & 0,004 & 2,003 \\
\hline Clupeidae & Sardina pilchardus & Walbaum, 1792 & 91 & 0,382 & 3,534 \\
\hline Gobiidae & Gobius cruentatus & Gmelin, 1789 & 1 & 0,004 & 0,091 \\
\hline Gobiidae & Gobius paganellus & Linnaeus, 1758 & 5 & 0,021 & 0,059 \\
\hline Labridae & Coris julis & Linnaeus, 1758 & 798 & 3,349 & 33,234 \\
\hline Labridae & Labrus merula & Linnaeus, 1758 & 1 & 0,004 & 0,852 \\
\hline Labridae & Labrus viridis & Linnaeus, 1758 & 5 & 0,021 & 2,447 \\
\hline Labridae & Sparisoma cretense & Linnaeus, 1758 & 5 & 0,021 & 0,527 \\
\hline Labridae & Symphodus cinereus & $\begin{array}{l}\text { Bonnaterre, } \\
1788\end{array}$ & 7 & 0,029 & 0,398 \\
\hline Labridae & Symphodus doderleini & Jordan, 1890 & 20 & 0,084 & 0,374 \\
\hline Labridae & $\begin{array}{l}\text { Symphodus } \\
\text { mediterraneus }\end{array}$ & Linnaeus, 1758 & 49 & 0,206 & 1,824 \\
\hline Labridae & $\begin{array}{l}\text { Centrolabrus } \\
\text { melanocercus }\end{array}$ & Risso, 1810 & 17 & 0,071 & 0,290 \\
\hline Labridae & Symphodus roissali & Risso, 1810 & 3 & 0,013 & 0,112 \\
\hline Labridae & Symphodus rostratus & Bloch, 1791 & 4 & 0,017 & 0,035 \\
\hline Labridae & Symphodus tinca & Linnaeus, 1758 & 214 & 0,898 & 11,304 \\
\hline Labridae & Thalassoma pavo & Linnaeus, 1758 & 979 & 4,109 & 46,125 \\
\hline Mugilidae & Chelon labrosus & Risso, 1827 & 10 & 0,042 & 1,107 \\
\hline Mugilidae & Mugil cepalus & Linnaeus, 1758 & 1 & 0,004 & 0,689 \\
\hline Mullidae & Mullus barbatus* & Linnaeus, 1758 & 20 & 0,084 & 1,536 \\
\hline Mullidae & Mullus surmuletus* & Linnaeus, 1758 & 51 & 0,214 & 3,436 \\
\hline Muraenidae & Muraena helena* & Linnaeus, 1758 & 6 & 0,025 & 3,918 \\
\hline
\end{tabular}




\begin{tabular}{|c|c|c|c|c|c|}
\hline Phycidae & Phycis phycis & Linnaeus, 1766 & 1 & 0,004 & 3,040 \\
\hline Pomacentridae & Chromis chromis & Linnaeus, 1758 & 17445 & 73,221 & 134,627 \\
\hline Sciaenidae & Sciaena umbra* & Linnaeus, 1758 & 3 & 0,013 & 3,976 \\
\hline Scorpaenidae & Scorpaena notata* & $\begin{array}{l}\text { Rafines- } \\
\text { que, } 1810\end{array}$ & 12 & 0,050 & 0,417 \\
\hline Scorpaenidae & Scorpaena porcus* & Linnaeus, 1758 & 2 & 0,008 & 0,229 \\
\hline Scorpaenidae & Scorpaena scrofa & Linnaeus, 1758 & 6 & 0,025 & 0,172 \\
\hline Serranidae & Anthias anthias & Linnaeus, 1758 & 982 & 4,122 & 16,085 \\
\hline Serranidae & Epinephelus costae* & $\begin{array}{l}\text { Steindachner, } \\
1878\end{array}$ & 2 & 0,008 & 6,971 \\
\hline Serranidae & $\begin{array}{l}\text { Epinephelus } \\
\text { marginatus* }\end{array}$ & Lowe, 1834 & 59 & 0,248 & 236,532 \\
\hline Serranidae & Mycteroperca rubra* & Bloch, 1793 & 2 & 0,008 & 6,985 \\
\hline Serranidae & Serranus cabrilla* & Linnaeus, 1758 & 43 & 0,180 & 2,900 \\
\hline Serranidae & Serranus scriba* & Linnaeus, 1758 & 91 & 0,382 & 7,728 \\
\hline Sparidae & Boops boops & Linnaeus, 1758 & 84 & 0,353 & 5,284 \\
\hline Sparidae & Dentex dentex* & Linnaeus, 1758 & 1 & 0,004 & 0,802 \\
\hline Sparidae & Diplodus annularis* & Linnaeus, 1758 & 33 & 0,139 & 3,809 \\
\hline Sparidae & Diplodus puntazzo* & $\begin{array}{l}\text { Walbaum [ex } \\
\text { Cetti], } 1792\end{array}$ & 9 & 0,038 & 2,119 \\
\hline Sparidae & $\begin{array}{l}\text { Diplodus } \\
\text { sargus sargus* }\end{array}$ & Linnaeus, 1758) & 87 & 0,365 & 10,527 \\
\hline Sparidae & Diplodus vulgaris* & $\begin{array}{l}\text { Geoffroy } \\
\text { Saint-Hilaire, } \\
1817\end{array}$ & 256 & 1,075 & 29,840 \\
\hline Sparidae & $\begin{array}{l}\text { Lithognathus } \\
\text { mormyrus* }\end{array}$ & Linnaeus, 1758 & 2 & 0,008 & 0,110 \\
\hline Sparidae & Oblada melanura & Linnaeus, 1758 & 76 & 0,319 & 7,239 \\
\hline Sparidae & Pagrus pagrus* & Linnaeus, 1758 & 3 & 0,013 & 0,712 \\
\hline Sparidae & Sarpa salpa & Linnaeus, 1758 & 433 & 1,817 & 54,058 \\
\hline Sparidae & Spicara maena & Linnaeus, 1758 & 148 & 0,621 & 10,141 \\
\hline Sparidae & Spicara smaris & Linnaeus, 1758 & 656 & 2,753 & 51,330 \\
\hline Sparidae & $\begin{array}{l}\text { Spondyliosoma } \\
\text { cantharus }\end{array}$ & Linnaeus, 1758 & 74 & 0,311 & 7,974 \\
\hline Sphyraenidae & $\begin{array}{l}\text { Sphyraena } \\
\text { viridensis } \times\end{array}$ & Cuvier, 1829 & 437 & 1,834 & 811,560 \\
\hline Synodontidae & Synodus saurus & Linnaeus, 1758 & 2 & 0,008 & 0,124 \\
\hline Trachinidae & Trachinus draco & Linnaeus, 1758 & 1 & 0,004 & 0,056 \\
\hline Tripterygiidae & Tripterygion delaisi & $\begin{array}{l}\text { Cadenat \& } \\
\text { Blache, } 1970\end{array}$ & 25 & 0,105 & 0,105 \\
\hline Total fam: 21 & Total sp: 56 & & $\begin{array}{l}\text { Total ab: } \\
23825\end{array}$ & $\begin{array}{l}\text { Total ab } \\
\text { rel: } 100\end{array}$ & Total bio: 1553,145 \\
\hline
\end{tabular}


El análisis PERMANOVA para la variable biomasa total de la comunidad íctica estudiada mostró cómo varía la comunidad de peces en función de la zona de protección, teniendo en cuenta la variabilidad espacial (localidad) y temporal (tiempo), de lo que se deduce que existe una variación clara por zonas entre las poblaciones de peces registrados en ellas. Las demás fuentes de variación no mostraron diferencias significativas, por lo que nos centraremos en las tres zonas de la reserva. En el análisis a posteriori de las comparaciones por pares (Pair-wise) se observaron diferencias significativas entre las zonas Integral y General (tabla 3).

Tanto para las especies comerciales bentónicas como para el mero (E. marginatus), se observó también una variabilidad espacial significativa dependiendo de la zona de la reserva. En el análisis a posteriori de las comparaciones por pares (Pairwise) se observó con más detalle una clara diferencia entre las zonas Parcial e Integral en ambos grupos, aunque también mostraron diferencias significativas entre las zonas General e Integral (tabla 3).

Por otro lado, en las especies comerciales pelágicas se observó una variabilidad espacial significativa dependiendo del tiempo de muestreo.

\begin{tabular}{|c|c|c|c|c|c|c|}
\hline \multicolumn{7}{|c|}{ A) PERMANOVA: Biomasa de la comunidad ictica } \\
\hline COMUNIDAD ÍCTICA & $\mathrm{df}$ & SS & MS & Pseudo-F & P (PERM) & Perms \\
\hline Zona & 2 & 26,225 & 13,113 & 2,2635 & 0,0314 & 4976 \\
\hline Tiempo & 1 & 4,4246 & 4,4246 & 1,5838 & 0,1826 & 4988 \\
\hline Localidad (zo) & 9 & 34,772 & 3,8636 & 1,3915 & 0,134 & 4974 \\
\hline Zona $\mathrm{x}$ Tiempo & 2 & 6,1566 & 3,0783 & 1,1189 & 0,3499 & 4984 \\
\hline Loc. (zona) x Tiempo & 9 & 24,989 & 2,7765 & 0,68235 & 0,9336 & 4975 \\
\hline Residual & 53 & 215,66 & 4,0691 & & & \\
\hline Total & 76 & 316,64 & & & & \\
\hline
\end{tabular}

\begin{tabular}{lccc}
\hline B) Pair-wise test & & & \\
\hline \multicolumn{1}{c}{ Comunidad íctica } & $\mathrm{t}$ & P (PERM) & Unique PERMs \\
\hline Parcial, General & 1,2437 & 0,1702 & 4987 \\
\hline Parcial, Integral & 1,778 & 0,132 & 3176 \\
\hline General, Integral & 1,5019 & $\mathbf{0 , 0 6 5 6}$ & 4983 \\
\hline
\end{tabular}




\begin{tabular}{lcccccc}
\hline \begin{tabular}{l} 
C) PERMANOVA: Biomasa de especies comerciales bentónicas \\
Especies comerCiALES \\
\multicolumn{1}{c}{ BENTÓNICAS }
\end{tabular} & df & SS & MS & PsEUDO-F & P (PERM) & PERMS \\
\hline Zona & 2 & 13,571 & 6,7855 & 3,2513 & $\mathbf{0 , 0 2 8 8}$ & 4989 \\
\hline Tiempo & 1 & 0,7598 & 0,7598 & 0,6483 & 0,5085 & 4986 \\
\hline Localidad (zo) & 9 & 15,329 & 1,7032 & 1,4548 & 0,2158 & 4979 \\
\hline Zona x Tiempo & 2 & 1,4085 & 0,7043 & 0,6154 & 0,6341 & 4988 \\
\hline Loc. (zona) x Tiempo & 9 & 10,536 & 1,1707 & 0,9238 & 0,5383 & 4981 \\
\hline Residual & 53 & 67,17 & 1,2674 & & & \\
\hline Total & 76 & 109,06 & & & & \\
\hline
\end{tabular}

\begin{tabular}{lccc}
\hline D) Pair-wise test & & & \\
\hline Especies Comerciales bentónicas & $\mathrm{t}$ & P (PERM) & Unique PERMs \\
\hline Parcial, General & 1,541 & 0,1134 & 4992 \\
\hline Parcial, Integral & 4,0774 & $\mathbf{0 , 0 2 7}$ & 3143 \\
\hline General, Integral & 1,6092 & $\mathbf{0 , 0 7 6 8}$ & 4988 \\
\hline
\end{tabular}

\begin{tabular}{|c|c|c|c|c|c|c|}
\hline \multicolumn{7}{|c|}{ E) PERMANOVA: Biomasa de especies comerciales pelágicas } \\
\hline $\begin{array}{c}\text { ESPECIES COMERCIALES } \\
\text { PELÁGICAS }\end{array}$ & df & SS & MS & Pseudo-F & P (PERM) & PERMS \\
\hline Zona & 2 & 8,7393 & 4,3696 & 2,1214 & 0,1478 & 4993 \\
\hline Tiempo & 1 & 2,6224 & 2,6224 & 4,5801 & 0,0486 & 4991 \\
\hline Localidad (zo) & 9 & 7,4192 & 0,8244 & 1,4721 & 0,249 & 4982 \\
\hline Zona x Tiempo & 2 & 2,9658 & 1,4829 & 2,6493 & 0,1162 & 4993 \\
\hline Loc (zona) x Tiempo & 9 & 5,0399 & 0,56 & 0,3718 & 0,9546 & 4978 \\
\hline Residual & 53 & 79,837 & 1,5064 & & & \\
\hline Total & 76 & 111,77 & & & & \\
\hline
\end{tabular}

\begin{tabular}{lcccccc}
\hline F) PERANOVA: Biomasa de Epinephelus marginatus & & & \\
\hline Epinephelus marginatus & $\mathrm{df}$ & SS & MS & Pseudo-F & P (PERM) & PERMS \\
\hline Zona & 2 & 12,575 & 6,2877 & 4,0513 & $\mathbf{0 , 0 3 3 8}$ & 4982 \\
\hline Tiempo & 1 & 0,551 & 0,551 & 0,69 & 0,4293 & 4948 \\
\hline Localidad (zo) & 9 & 11,675 & 1,2953 & 1,6258 & 0,235 & 4992 \\
\hline Zona x Tiempo & 2 & 0,842 & 0,421 & 0,5471 & 0,5901 & 4987 \\
\hline Loc (zona) x Tiempo & 9 & 7,17 & 0,7967 & 0,8473 & 0,5877 & 4981 \\
\hline Residual & 53 & 49,831 & 0,9402 & & & \\
\hline Total & 76 & 82,981 & & & & \\
\hline
\end{tabular}




\section{COMUNIDAD ÍCTICA}

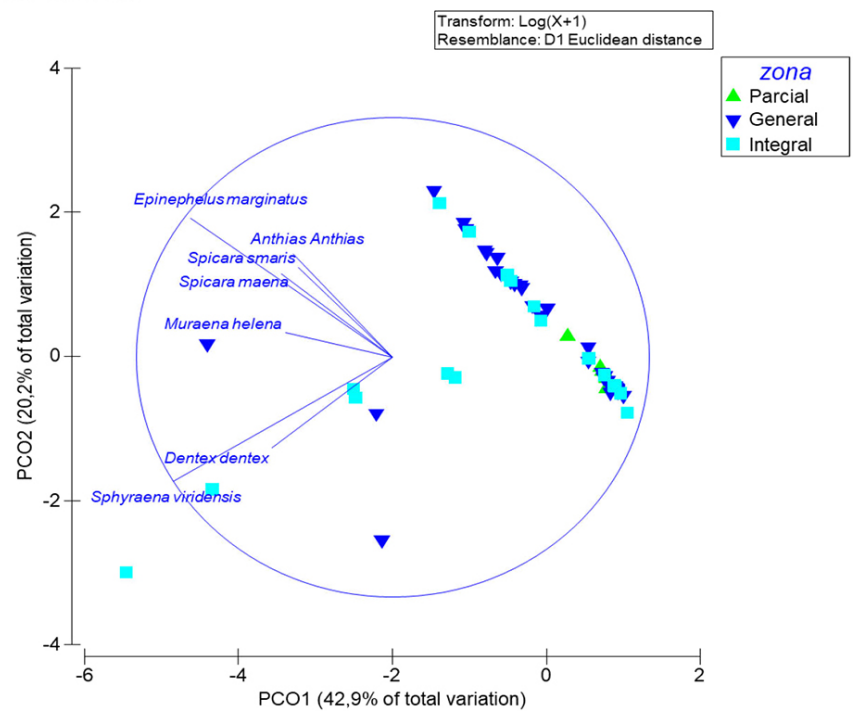

Figura 12. Análisis por ordenación de componentes principales (PCO) para la biomasa total de la comunidad de peces. Los vectores señalan la distribución de especies registradas en los muestreos realizados y el coeficiente de correlación según el tamaño cada ejemplar.

\begin{tabular}{lccc}
\hline G) Pair-wise test & & & \\
\hline Epinephelus marginatus & $\mathrm{t}$ & P (PERM) & UNIQUE PERMs \\
\hline Parcial, General & 1,7362 & 0,1104 & 4987 \\
\hline Parcial, Integral & 5,9607 & $\mathbf{0 , 0 2 1 6}$ & 3165 \\
\hline General, Integral & 1,7324 & 0,1162 & 4989 \\
\hline
\end{tabular}

El PCO para toda la comunidad de peces muestra claramente una distribución homogénea situada en la parte derecha del gráfico y caracterizada por la presencia de la gran mayoría de las especies a excepción de E. marginatus, A. anthias, $S$. maena, S. smaris, $M$. helena, D. dentex y $S$. viridensis, situadas en las zonas Integral y General en la parte izquierda del gráfico (figura 12).

El PCO para la biomasa total de la comunidad de especies comerciales bentónicas mostró una distribución homogénea de los muestreos (figura 13).

En el caso de las especies comerciales pelágicas estas mostraron variabilidad espacial significativa de las poblaciones de peces comerciales bentónicos dependiendo del factor tiempo, que varió entre los meses de junio y julio a agosto y septiembre.

El resultado de hacer el análisis de ordenación de componentes principales (PCO) para la biomasa total de la comunidad de especies comerciales pelágicas fue 


\section{BENTÓNICAS}

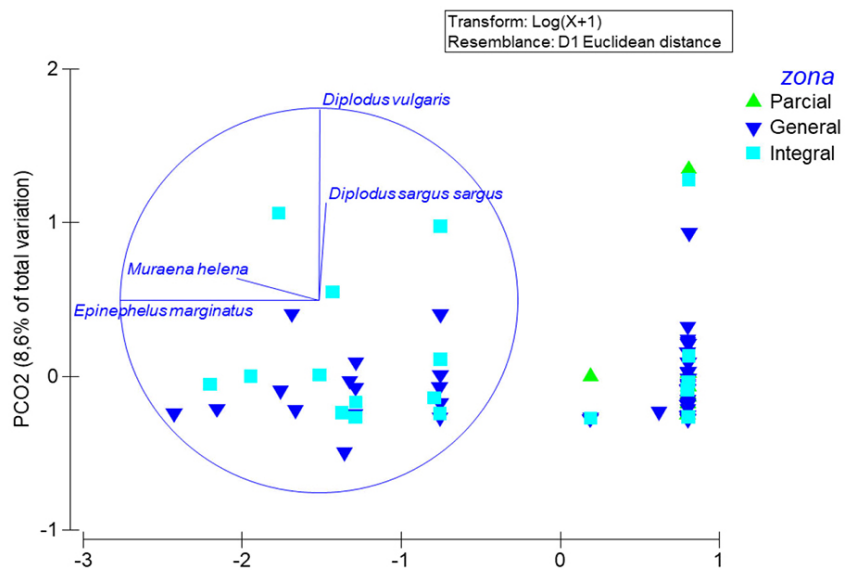

Figura 13. Análisis por ordenación de componentes principales (PCO) para la biomasa de la comunidad de especies comerciales bentónicas. Los vectores señalan la distribución de especies registradas en los muestreos realizados y el coeficiente de correlación según el tamaño cada ejemplar.

\section{COMUNIDAD SP COMERCIALES} PELÁGICAS

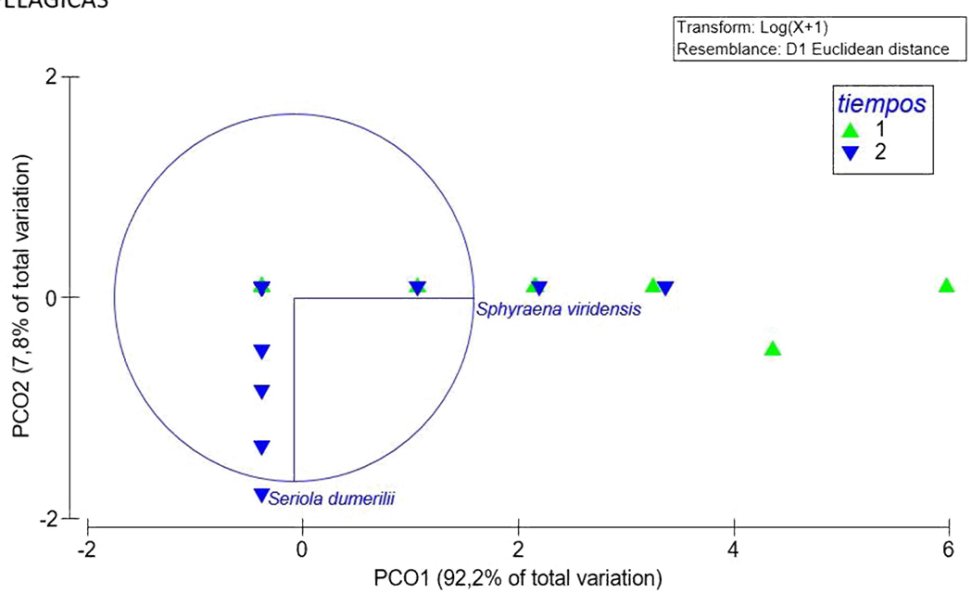

Figura 14. Análisis por ordenación de componentes principales (PCO) para la biomasa de la comunidad de especies comerciales pelágicas. Los vectores señalan la distribución de especies registradas en los muestreos realizados y el coeficiente de correlación según el tamaño cada ejemplar. 


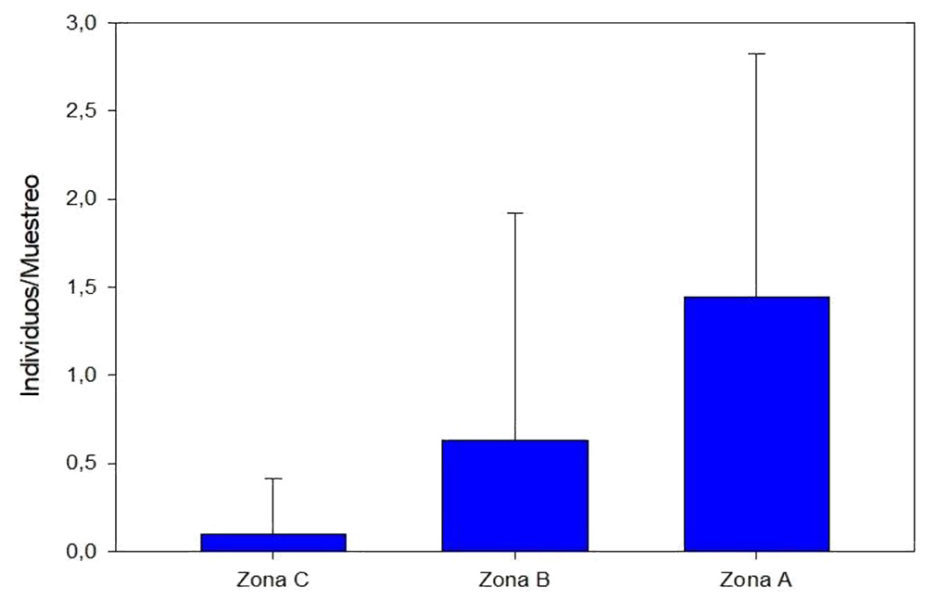

Niveles de protección de la reserva marina de las de Ventotene y Santo Stefano

Figura 15. Gráfico de barras de la especie Epinephelus marginatus que indica el número medio de ejemplares por muestreo en los distintos niveles de protección de la reserva marina de VSS.

una clara separación de los muestreos donde hacia la derecha se observa el tiempo 1 que se corresponde con los meses de junio y julio y hacia la izquierda los muestreos del tiempo 2 de agosto y septiembre. Los muestreos del tiempo 1 estuvieron caracterizados por la presencia de $S$. viridensis, mientras que los del tiempo 2 estuvieron caracterizados por $S$. dumerili (figura 14).

Por último, la especie E. marginatus mostró diferencias significativas entre zonas. En el análisis a posteriori de las comparaciones por pares (Pair-wise) se observó una clara diferencia entre las zonas Parcial e Integral (figura 15).

\section{DISCUSIÓN}

Gracias a los resultados obtenidos hemos podido comprobar que existe un claro efecto reserva en el Área Marina Protegida de las islas de Ventotene y Santo Stefano. Específicamente, se observó un aumento de la abundancia de especies emblemáticas y de interés comercial al aumentar el nivel de protección. Por lo tanto, podemos decir que este AMP cumple, en ese aspecto, con la finalidad esperada.

Este es otro ejemplo más de cómo las AMP juegan un papel importante al actuar como áreas de refugio y reabastecimiento para las poblaciones objetivo de pesca y, por lo tanto, debe ser un objetivo principal de quienes las manejan, desarrollar el máximo potencial en la conservación que ofrecen estas áreas protegidas. 
Debido a la limitación de las actividades de pesca dentro del AMP, y especialmente dentro de la zona integral, los efectos beneficiosos esperados incluyen un aumento de la biodiversidad, la abundancia y el tamaño de las especies marinas (Russ, 2002; Tetreault \& Ambrose, 2007). El desarrollo de un AMP para la obtención de un buen «efecto reserva» depende en una gran medida del tamaño (Claudet et al., 2008) y, a pesar de que el AMP de éstas Islas no es muy extenso, se ha comprobado que pequeñas reservas han dado lugar a grandes aumentos en el tamaño de los peces, especialmente en especies comerciales sedentarias ya que la mayor parte de su área de distribución está pensada para ser incluido dentro de estas áreas (Kramer \& Chapman, 1999; (García-Charton et al., 2004; Claudet et al., 2008). Además, algunas especies como las demersales de libre movimiento muestran respuestas positivas con respecto a la densidad cuando están protegidas (Claudet et al., 2010; Abecasis et al., 2015).

En nuestro caso, el recuento de la abundancia y biomasa junto con el análisis estadístico mostró claramente cómo la comunidad de peces varió significativamente dependiendo de la zona de protección en la que se encuentren, siendo la zona general y la integral las dos más distintas en cuanto a biomasa de especies. Este hecho engloba a todos los tipos de peces encontrados, por lo que podemos generalizar que el nivel tan alto de protección en la zona integral sea el factor clave, puesto que la zona de reserva general y reserva parcial comparten muchas similitudes a la hora de las restricciones del reglamento.

En el caso de las especies comerciales bentónicas, podemos afirmar también que a mayor grado de protección de la reserva, hay una mayor abundancia y biomasa de estas especies. En los estudios realizados se observa una diferencia significativa entre las zonas de reserva integral A y reserva parcial C, debida a la navegación y el efecto antrópico que conlleva cada una; el fondeo, que provoca la fragmentación del hábitat, y la prohibición de la pesca. Estas actividades actúan indirectamente con la comunidad de peces, quedando este hecho reflejado en los resultados.

Otro factor importante dentro de la comunidad de peces que incluye esta AMP es la amplia biomasa de especies pelágicas de gran interés comercial. Una explicación podrían ser las grandes zonas de no captura que pueden permitir un mayor número de peces móviles con amplios rangos de hogar para permanecer protegidos dentro de la reserva marina, en comparación con los más pequeños (Chapman $\&$ Kramer, 2000; Jennings, 2001, Apostolaki et al., 2002; Claudet et al., 2008). En el caso de Sphyraena viridensis, observamos como su biomasa fue mayor sobre todo en los muestreos realizados en junio y julio. La explicación estaría relacionada con la temperatura. Tal y como cuentan Fontes y Afonso (2017), existe una migración de esta especie con la estación del ańo, siendo junio y julio los meses de verano con el agua más fría los elegidos por esta especie para aparecer por la zona. Por otro lado, la otra especie pelágica de interés que se ha investigado en este estudio, Seriola dumerili, presenta una amplia distribución en el tiempo 2, en los meses de agosto a septiembre, por lo que los cambios de temperatura de $24^{\circ} \mathrm{C}$ a $28^{\circ} \mathrm{C}$ podrían haber afectado a la migración de estas especies (García-Gómez, 1993; Mazzola et al., 2000).

El análisis de los datos recopilados durante el censo de peces de las islas de Ventotene y Santo Stefano permite numerosas observaciones que, sin embargo, deben 
evaluarse con precaución. La fauna de peces se caracteriza por su propia naturaleza y por una alta variabilidad temporal. Para poder extraer indicaciones más sólidas, sería necesario continuar las campañas a lo largo de los años para poder evaluar las tendencias temporales y la construcción de un diseño de muestreo no limitado por los recursos disponibles para su implementación.

Por último, cabe destacar la importancia y protección que tiene el mero oscuro (Epinephelus marginatus) como especie emblemática y de interés comercial. Dado que su pesca está prohibida en toda la reserva (http://www.riservaventotene.it), esta especie puede crecer libremente y alcanzar longitudes de más de un metro, sobre todo en la Zona A, donde hemos observado un aumento considerable de la biomasa media. No es raro observar en la reserva integral muchos ejemplares de gran tamaño mientras que se encuentras pocos individuos y de tamaño reducido en la Zona $\mathrm{C}$ de reserva parcial. Estos resultados también indican que a pesar de que su pesca causa el mayor impacto sobre sus poblaciones, otras actividades como las permitidas en otras zonas del AMP pueden estar afectando a sus poblaciones, ya que presentan menores biomasas.

La comparación con otros estudios ya publicados de AMPs del Mediterráneo nos permite decir que las islas de Ventotene y Santo Stefano presentan una gran diversidad íctica. De manera más detallada, Claudet et al., en el 2006 censaron unas 40 especies de peces; Seytre y Francour en el 2008, 46 especies o Tunesi et al., en el 2006, con 48. En nuestro caso, las islas de Ventotene y Santo Stefano con un total de 56 especies censadas (tabla especies), parecen ser un hot spot de biodiversidad en el Mediterráneo. A pesar de que la zona de reserva integral es la más pequeña, existe una biodiversidad de peces muy grande, por lo que podemos decir que no afecta tanto el tamaño como el nivel de protección o el diseño de la reserva (Gurevitch \& Hedges, 1999; Claudet et al., 2008). Además, resaltar que durante este estudio se han registrado dos especies más que el estudio realizado anteriormente en el 2010, en esta misma área marina protegida, por Orio (2010).

\section{AGRADECIMIENTOS}

Me gustaría agradecer a las personas que me han ayudado a realizar este trabajo durante todo este tiempo. En primer lugar, a mi tutor José Carlos Hernández por su interés, dedicación y paciencia a lo largo de estos meses.

Al equipo del Diving World Ventotene, y en particular a Valentina Lombardi por su completa disponibilidad, conocimientos, consejos y apoyo para cualquier aspecto del trabajo.

Al director de la reserva marina protegida de Ventotene y Santo Stefano, Antonio Romano, por su permiso a la hora de realizar los muestreos dentro de la reserva.

Al director del máster, Alberto Brito, por su inagotable paciencia, sabiduría y su buena predisposición siempre.

Por último, me gustaría agradecer a mi amigo Alessandro Orio, por su ayuda, conocimientos y estudios acerca de ésta AMP en particular. 
Sin más, espero que a todos los lectores les haya resultado interesante mi trabajo.

\section{CONTRIBUCIÓN DE LOS AUTORES}

The author is the only responsible for the whole paper Dr. José Carlos Hernández helped with the data analysis.

RECIBIDO: agosto de 2019; ACEPTADo: enero de 2020 


\section{REFERENCIAS}

Abecasis, D., Horta e Costa, B., Afonso, P., Gonçalves, E.J. \& Erzini, K. 2015. Early reserve effects linked to small home ranges of a commercial fish, Diplodus sargus, Sparidae. Marine Ecology Progress Series, 518: 255-266.

Anderson, M.J. 2005. Permanova: A fortran computer program for permutational multivariate analysis of variance. Departament of Statistics. University of Auckland.

Apostolaki, P., Milner-Gulland, E.J., McAllister, M.K. \& Kirkwood, G.P. 2002. Modelling the effects of establishing a marine reserve for mobile fish species. Canadian Journal of Fisheries and Aquatic Sciences, 59(3): 405-415.

Badalamenti, F., Ramos, A.A., Voultsiadou, E., Sánchez Lizaso, J.L., D’Anna, G., Pipitone, C., Mas, J., Ruiz Fernández, J.A., Whitmarsh, D. \& Riggio, S. 2000. Cultural and socio-economic impacts of Mediterranean marine protected areas. Environmental Conservation, 27(2): 110-125.

BARDACH, J.E. 1959. The summer standing crop of fish on a shallow Bermuda reef. Limnology and Oceanography, 4: 77-85.

BELL, J.D. 1983. Effects of depth and marine reserve fishing restrictions on the structure of a rocky reef fish assemblage in the north-western Mediterranean Sea. Journal of applied ecology, 357-369.

Bortone, S.A., Kimmel, J.J. \& Bundrick, C.M. 1989. A comparison of three methods for visually assessing reef fish Effects of depth and marine reserve fishing restrictions on the structure of a rocky reef fish assemblage in the north-western Mediterranean Sea communities: time and area compensated. Gulf of Mexico Science, 10(2): 2.

Bortone, S.A., Van Tassel, J., Brito, A., Falcón, J.M. \& Bundrick, C.M. 1991. A visual assessment of the inshore fishes and sishery resources off El Hierro, Canary Islands: A baseline survey. Scientia Marina, 55(3): 529-541.

BRock, V.E., 1954. A preliminary report on a method of estimating reef fish populations. The Journal of Wildlife Management, 18: 297-308.

Cattaeo-Vietti, R., Guidetti, P., DiLorenzo, M. \& Bussotti, S. 2011. Pre-valutazione del "Effetto Riserva presso i cinque parchi marini della Liguria. Centro di Ricerca in Biologia Marina del Mar Ligure (BIOLMAR), Università di Genova, 152 pp.

Chapman, M.R. \& Kramer, D.L. 2000. Movements of fishes within and among fringing coral reefs in Barbados. Environmental Biology of Fishes, 57(1): 11-24.

Claudet, J., Pelletier, D., Jouvenel, J.Y., Bachet, F. \& Galzin, R. 2006. Assessing the effects of marine protected area (MPA) on a reef fish assemblage in a northwestern Mediterranean marine reserve: Identifying community-based indicators. Biological Conservation, 130: 349-369.

Claudet, J., Osenberg, C.W., Benedetti-Cecchi, L., Domenici, P., García-Charton, J.A., Pérez-Ruzafa, A., Badalamenti, F., Bayle-Sempere, J., Brito, A., Bulleri, F., Culioli, J.M., Dimech, M., Falcón, J.M., Guala, I., Milazzo, M., Sánchez-Meca, L., Somerfield, P., Stobart, B., Vandeperre, F., Valle, C. \& Planes, S. 2008. Marine reserves: Size and age do matter. Ecology Letters, 11: 481-489.

Claudet, J., Osenberg, C.W., Domenici, P., Badalamenti, F., Milazzo, M., Falcón, J., Bertocci, I., Benedetti-Cecchi, L., García-Charton, J.A., Goñi, R., Borg, J.A., Forcada, A., de Lucia, G., Pérez-Ruzafa, A., Afonso, P., Brito, A., Guala, I., Le Diréach, L., 
Sanchez-Jerez, P., Somerfield, P.J. \& Planes, S. 2010. Marine reserves: Fish life history and ecological traits matter. Ecological Applications, 20: 830-839.

Côté, I.M., Mosqueira, I. \& Reynolds, J.D. 2001. Effects of marine reserve characteristics on the protection of fish populations: A meta-analysis. Journal of Fish biology, 59: 178-189.

Di Donato, R. 2008. Sviluppo di basi di dati GIS e procedure d'analisi multi criterio per la pianificazione e la gestione di Aree Marine Protette. Tesi di Dottorato in Scienze ed Ingegneria del Mare, Università «Parthenope» di Napoli.

Di Stefano, F. 2004. Caratterizzazione ecologica e biogeografica del benthos di substrato solido nelle riserve marine di «Punta Campanella» e delle «Isole di Ventotene e Santo Stefano». Tesi di Dottorato di Ricerca in Biologia Evoluzionistica. Università degli Studi di Catania.

Fontes, J. \& Afonso, P. 2017. Long-term residency and movements of yellowmouth barracuda (Sphyraena viridensis) at a shallow seamount. Marine Biology, 164.

Francour, P. 1994. Pluriannual analysis of the reserve effect on ichthyofauna in the Scandola natural reserve (Corsica, Northwestern Mediterranean). Oceanologica Acta, 17: 309-317.

Francour, P., Ganteaume, A. \& Poulain, M. 1999. Effects of boat anchoring in Posidonia oceanica seagrass beds in the Port-Cros National Park (north-western Mediterranean Sea). Aquatic Conservation: Marine Freshwater Ecosystem, 9: 391-400.

Francour, P., Harmelin, J.G., Pollard, D. \& Sartoretto, S. 2001. A review of marine protected areas in the northwestern Mediterranean region: siting, usage, zonation and management. Aquatic Conservation: Marine Freshwater Ecosystems, 11: 155-188

Fraschetti, S., Terlizzi, A., Bussotti, S., Guarnieri, G., D’Ambrosio, P. \& Boero, F. 2005. Conservation of Mediterranean seascapes: analyses of existing protection schemes. Marine Environmental Research, 59: 309-332.

García-Charton, J.A., Pérez-Ruzafa, A., Sánchez-Jérez, P., Bayle-Sempere, J.T., Reñones, O. \& Moreno, D. 2004. Multi-scale spatial heterogeneity, habitat structure, and the effect of marine reserves on Western Mediterranean rocky reef fish assemblages. Marine Biology, 144: 161-182.

García-Charton, J.A., Pérez-Ruzafa, A., Marcos, C., Claudet, J., Badalamenti, F., Benedetti-Cecchi, L., Falcón, J.M., Milazzo, M., Schembri, P.J., Stobart, B., Vandeperre, F., Brito, A., Chemello, R., Dimech, M., Domenici, P., Guala, I., Le Diréach, L., Maggi, E. \& Planes, S. 2008. Effectiveness of European Atlanto-Mediterranean MPAs: Do they acomplish the expected effects on populations, communities and ecosystems?. Journal for Nature Conservation, 16: 193-221.

García-Gómez, A., 1993. Primeras experiencias de crecimiento de juveniles de Seriola mediterranea (Seriola dumerili, Risso, 1810), alimentados con una dieta semihumeda. Boletín del Instituto Español de Oceanografía, 9(2): 347-360.

García-Rubies, A. \& Zabala, M. 1990. Effects of total fishing prohibition on the rocky fish assemblages of Medes Islands marine reserve (NW Mediterranean). Scientia Marina, 54: 317-328.

Guidetti, P., 2002. Mediterranean MPAs: the importance of experimental design in detecting the effects of protection measures on fish. Aquatic Conservation: Marine and Freshwater Ecosystems, 12: 619-634.

Guidetti, P., Bussotti, S. \& Boero, F., 2005. Evaluating the effects of protection on fish predators and sea urchins in shallow artificial rocky habitats: a case study in the northern Adriatic Sea. Marine Environmental Research, 59: 333-348. 
Gurevitch, J. \& Hedges, L.V. 1999. Statistical issues in ecological meta-analyses. Ecology, 80(4): $1142-1149$

Harmelin-Vivien, M. \& Harmelin, J.G., 1975. Presentation d'une methode d'evaluation «in situ» de la faune ichtyologique. Travaux Scientifiques du Parc national de Port-Cros, 1: 47-52.

Harmelin, J.G., Bachet, F. \& Garcia, F. 1995. Mediterranean marine reserves: fish indices as tests of protection efficiency. Marine Ecology, 16: 233-250.

Jackson, J.B., Kirby, M., Berger, W., Karen A., Bjorndal, K., Botsford, L., Bourque, B., Bradbury, R., Cooke, R., Erlandson, J., Estes, J., Hughes, T., Kidwell, S., Lange, C., Lenihan, H., Pandolfi, J., Peterson, C., Steneck, R., Tegner, M. \& Warner, R. 2001. Historical overfishing and the recent collapse of coastal ecosystems. Science, 293: 629-638.

Jennings, S. 2001. Patterns and prediction of population recovery in marine reserves. Reviews in Fish Biology and Fisheries, 10(2): 209-231.

Kramer, D.L. \& Chapman, M.R. 1999. Implications of fish home range size and relocation for marine reserve function. Environmental Biology Fishes, 55: 65-79.

Mapstone, B. \& Ayling, A. 1998. An Investigation of optimum methods and unit sizes for the visual estimation of abundances of some coral reef organisms. Great Barrier Reef Marine Park Authority.

Marino, D. (1810) 2011. Le aree marine protette italiane. Stato.

Massaro, G. 2009. Metodi di gestione delle Aree Marine Protette: attività di ricerca per l'applicazione di tecniche ed indicatori di efficacia. Tesi di Dottorato di Ricerca in Scienze Ambientali, Università Ca' Foscari, Venezia.

Mazzola, A., Favaloro, E. \& Gianluca, S. 2000. Cultivation of the Mediterranean amberjack, Seriola dumerili (Risso, 1810), in submerged cages in the Western Mediterranean Sea. Aquaculture, 181: 257-268.

Mosqueira, I., Côté, I.M., Jennings, S. \& Reynolds, J. 2000. Conservation benefits of marine reserves for fish populations. Animal Conservation, 4: 321-332.

Orio, A. 2001. Analisi dei popolamenti ittici nell'Area Marina Protetta di Ventotene e Santo Stefano attraverso la metodologia Underwater Visual Census. Tesi di Laurea Magistrale in Scienze del Mare. Università di Roma.

Reñones, O., Poulin, N.V. \& Goni, R. 2005. Size related dietary shifts of Epinephelus marginatus in a western Mediterranean littoral ecosystem: an isotope and stomach content analysis. Journal of Fish Biology, 61: 122-137.

Russ, G.R. 2002. Yet another review of marine reserves as reef fishery management tools. Coral Reef Fishes: Dynamics and Diversity in a Complex Ecosystem, 421-443.

Sánchez-Lizaso, J.L., Goni, R., Reñones, O., Garcia-Charton, A., Galzin, R., Bayle, J.T., Sanchez-Jerez, P., Perez Ruzafa, A. \& Ramos, A. 2000. Density dependence in marine protected populations: a review. Environmental conservation, 27(2): 144-158.

Shears, N.T. \& Babcock, R.C. 2002. Marine reserves demonstrate top-down control of community structure on temperate reefs. Oecologia, 132: 131-142.

Seytre, C. \& Francour, P. 2008. Is the Cape Roux marine protected area (Saint-Raphael, Mediterranean Sea) an efficient tool to sustain artisanal fisheries? First indications from visual censuses and trammel net sampling. Aquatic Living Resources, 21: 297-305. 
Tegner, M.J. \& Dayton, P.K. 2000. Ecosystem effects of fishing in kelp forest communities. Journal of Marine Science, 57: 579-589.

Tetreault, I. \& Ambrose, R.F. 2007. Temperate marine reserves enhance targeted but not untargeted fishes in multiple no-take MPAs. Ecological Applications, 17: 2251-2267.

Tunesi, L. \& Diviacco, G. 1993. Environmental and socio-economic criteria for the establishment of marine coastal parks. International Journal of Environmental Studies, 43: 253-259.

Tunesi, L., Molinari, A. \& Salvati, E. 2006. Fish assemblage of the marine protected area of Cinque Terre (NW Mediterranean Sea): First characterization and assessment by visual census. Chemistry and Ecology, 22(4): 245-253.

Worm, B., Barbier, E., Beaumont, N., Duffy, J., Folke, C., Halpern, B., Jackson, J., Lotze, H., Micheli, F., Palumni, S., Sala, E., Selkoe, K., Stachowicz, J. \& Watson, R. 2006. Impacts of biodiversity loss on ocean ecosystem services. Science, 314: 787-790. 